\section{Hetrogeneous Integration and Fabrication of III-V MOS Devices in a 200mm Processing Environment}

Niamh Waldron, Duy Nguyen ${ }^{1}$, Dennis Lin, Guy Brammertz, Benjamin Vincent, Andrea Firrincieli², Gillis Winderick, Sonja Sioncke, Brice de Jaeger, Gang Wang, Jerome Mitard, Wei-E Wang, Marc Heyns ${ }^{2}$, Matty Caymax, Marc Meuris, Philippe Absil and Thomas Y. Hoffman

Imec, Kapeldreef 75, B-3001 Leuven, Belgium also at ${ }^{1}$ Université de Liège, Institut de Physique B5a, Allée du 6 Août17, B-4000 Liège,Belgium ${ }^{2}$ Department of Metallurgy and Materials Engineering, K.U. Leuven, 3000 Leuven, Belgium

As CMOS continues to scales to more advanced nodes, new higher mobility channel materials have been considered as an alternative to $\mathrm{Si}$ in order to meet power and performance requirements [1]. Various III-V materials have recently emerged as an attractive option for nMOS. However, from an economical and technological standpoint it is imperative to implement III$\mathrm{V}$ devices in a Si CMOS fabrication environment in order to leverage the advantages of both large scale wafers and state-of-the-art Si equipment. The integration challenges of introducing III-V into a $\mathrm{Si}$ line include safety risk assessments from toxic materials, maintenance of tools after processing III-V, cross-contamination from hightemperature and wet etch steps, and modifying standard recipes wherever bare III-V materials are exposed during process. In this work we demonstrate the feasibility of processing III-V virtual substrates on a $\mathrm{Si}$ platform following a standard CMOS based approach without cross-contamination from the III-V.

The test vehicle used in this study is an n-type capacitor. It is processed in our standard $200 \mathrm{~mm}$ Si pilot line on virtual $200 \mathrm{~mm}$ GaAs substrates. These substrates are prepared by growing a 300nm n-type doped GaAs on a Ge-on-Si template (Fig 1). The details of the GaAs growth are described elsewhere [2]. After GaAs epi growth, windows are opened in a deposited oxide and the gate stack is deposited and patterned. Following spacer processing, topside contact to the GaAs is made by a novel self-aligned $\mathrm{Ge} / \mathrm{NiGe}$ scheme. A contact resistance of $0.13 \Omega . \mathrm{cm}$ was achieved using this approach. Device

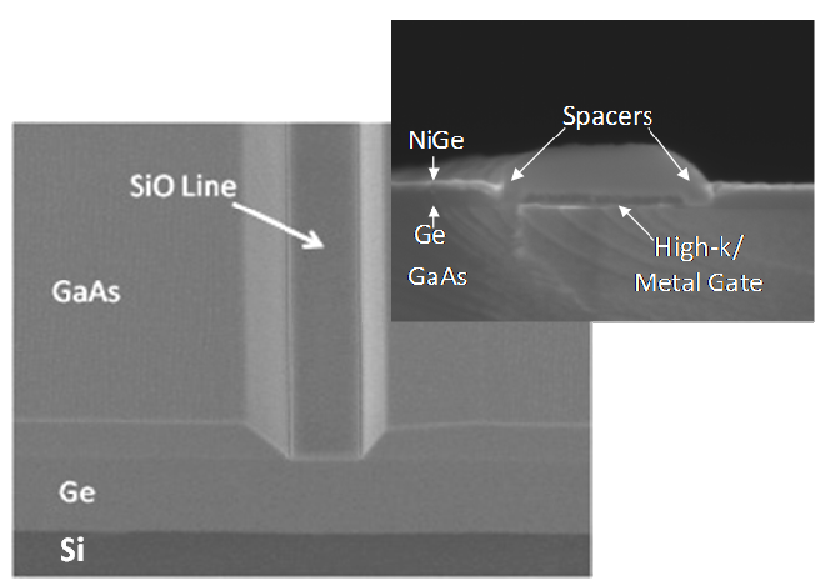

Fig 1: Tilted XSEM view of GaAs selective growth on GOS substrate. inset shows capacitor structure fabricated on the GaAs layer using a selective Ge/NiGe contacting scherne. fabrication is completed by standard W contact plug and $\mathrm{Cu}$ Metal 1 processing.

Capacitors ranging in size from $10 \mu \mathrm{m} \times 10 \mu \mathrm{m}$ to $100 \mu \mathrm{m}$ x $100 \mu \mathrm{m}$ were fabricated. The capacitance was found to be scalable and repeatable across the wafer. The CV curves obtained showed a frequency dispersion typical of GaAs capacitors without non-optimzed passivation. The optimization of the surface passivation is the subject of another study. In addition, the interfacial defect density Dit extracted from conudctance measurements, carried out at $25{ }^{\circ} \mathrm{C}$ and $150{ }^{\circ} \mathrm{C}$, were also very consistent with those of bulk GaAs samples previously measured (Fig. 2). This illustrates the intrinsic properties of the $200 \mathrm{~mm}$ GaAs substrates.

Within wafer and tool cross-contaminations are expected from III-V materials when they are integrated in a $\mathrm{Si}$ process flow. In this work we have performed a cross contamination assessment for both thermal and wet processing by means of TXRF monitoring and have outlined guides line that allow processing of III-V materials in a Si environment. We have found that the exposed GaAs surface does not suffer from any outgassing issue if the thermal processing is carried out at $300{ }^{\circ} \mathrm{C}$ or below. It will also be shown that with the correct choice of chemicals and tools for wet processing the risk of cross-contamination in these steps can be minimized.

In conclusion, $200 \mathrm{~mm}$ virtual III-V substrates were successfully run entirely through a Si processing line. The resulting electrical results were comparable to those of bulk GaAs. A CMOS compatible contacting scheme was developed. Guidelines have been set up to avoid crosscontamination. The results reported support a path to production for large scale III-V device wafers.

ACKNOWLEDGEMENT: The authors acknowledge the European Commission for finanacial support in the DualLogic project no. 214579. Further, we thank the imec core partners with the IIAP on Logic-Dram.

REFERENCES: [1]. Khakifirooz, TED 55, p1401, 2008 [2] Nguyen, ECS 2010

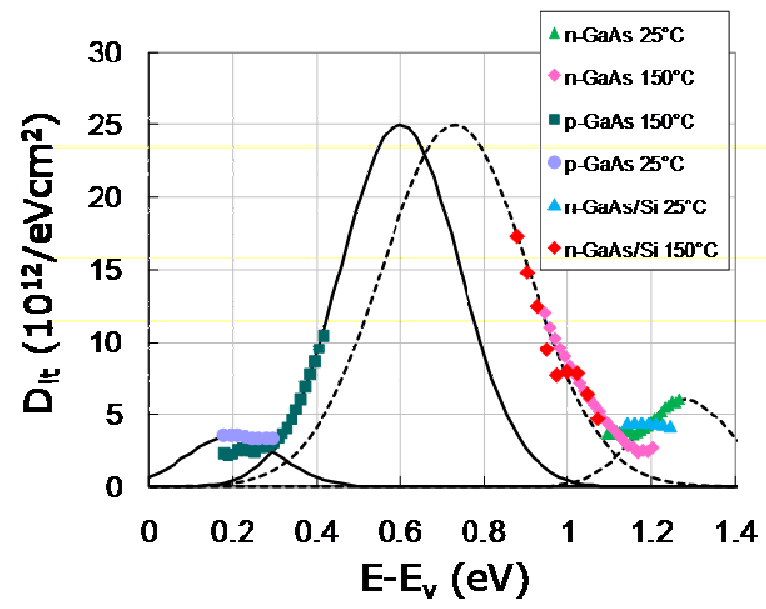

Fig 2: Extracted $D_{k}$ of the fabricated n-type GaAs capacitors measured at $25^{\circ} \mathrm{C}$ and $150^{\circ} \mathrm{C}$ compared to those of bulk GaAs samples with a similar passivation treatment. 